\title{
A Modular Method for Evaluating the Performance of Picture Archiving and Communication Systems
}

\author{
William H. Sanders, Latha A. Kant, and Abhijit Kudrimoti
}

\begin{abstract}
Modeling can be used to predict the performance of picture archiving and communication system (PACS) configurations under various load conditions at an early design stage. This is important because choices made early in the design of a system can have a significant impact on the performance of the resulting implementation. Because PACS consist of many types of components, it is important to do such evaluations in a modular manner, so that alternative configurations and designs can be easily investigated. Stochastic activity networks (SANs) and reduced base model construction methods can aid in doing this. SANs are a model type particularly suited to the evaluation of systems in which several activities may be in progress concurrently, and each activity may affect the others through the results of its completion. Together with SANs, reduced base model construction methods provide a means to build highly modular models, in which models of particular components can be easily reused. In this article, we investigate the use of SANs and reduced base model construction techniques in evaluating PACS. Construction and solution of the models is done using UltraSAN, a graphic-oriented software tool for model specification, analysis, and simulation. The method is illustrated via the evaluation of a realistically sized PACS for a typical United States hospital of 300 to $\mathbf{4 0 0}$ beds, and the derivation of system response times and component utilizations.
\end{abstract}

Copyright $\odot 1993$ by W.B. Saunders Company

KEY WORDS: performance evaluation, picture archiving and communication systems, stochastic Petri nets, stochastic activity networks.

COMPLETELY AUTOMATED networkbased systems for the generation, storage, transportation, processing, and presentation of digitized medical images in a hospital are expected to be an integral part of all hospital management systems in the next few years. These systems are called picture archiving and communication systems (PACS). PACS were

From the Department of Electrical and Computer Engineering, The University of Arizona, Tucson, AZ.

Supported in part by a grant from Medical Systems Division, Toshiba Corporation, and the Digital Equipment Corporation Faculty Program: Incentives for Excellence.

Address reprint requests to William $H$. Sanders, $P h D$, Department of Electrical and Computer Engineering, The University of Arizona, Tucson, AZ 85721

Copyright 1993 by W.B. Saunders Company

0897-1889/93/0603-0002\$3.00/0 developed to offer the advantages of better image accessibility, reduced overhead costs of image handling and improved diagnostic image quality. However, the huge amount of data handling and processing involved in PACS lead to problems related to limited storage and long processing and network delays, which may cause unacceptable response times to image viewing requests.

Model-based evaluation can be used to identify such problems and to investigate potential solutions in the early design phase of a project. If successful, such studies can aid in producing better system designs in a shorter period than would be possible if prototyping were the sole evaluation method used. In particular, we can predict the performance of PACS under various conditions by varying different parameter values in the simulation, eg, workloads and hardware specifications. Furthermore, simulation can be used to evaluate the algorithms and software processes that constitute the image processing aspect of PACS, eg, the image compression and image prefetching algorithms. The simulation of these algorithms and processes can then be used as a starting point for their implementation.

In this article, we investigate the use of stochastic activity networks (SANs) ${ }^{3,4}$ and reduced-base model construction methods ${ }^{5}$ in constructing models of PACS. SANs are used to build models of PACS components (ie, modalities, viewing workstations, networks, and database archives), and the reduced base model construction formalism is used to connect these components into different system configurations. This combination allows us to build models that are modular in the sense that many different configurations can be investigated by changing the interconnection of previously constructed component models. Construction and solution of the models is done using UltraSAN, ${ }^{6}$ a graphical software tool that aids in the construction and solution of systems represented as SANs.

To illustrate the use of these methods, we consider a comprehensive model of a complete 
PACS intended for a typical United States hospital of 300 to 400 beds. The PACS consists of three different types of imaging modalities ( $\mathrm{x}$-ray, ultrasound, and magnetic resonance imaging [MRI]) with several units of each type, a central database with short-term storage and long-term archiving facilities, and a cluster of workstations for image viewing. The various system components are connected together by a high-speed network. We have modeled the image generation process at a modality, the transmissions of new image folders to the database, the image migration algorithm in the database, the database operating system scheduling, and the processing of viewing requests from a workstation. We use the model to estimate the expected response times of viewing requests made at a workstation, the portion of this time due to database processing, the use of the communication network, and the uses of the hard disk and optical disk in the database. We also investigate a system configuration in which all modalities are of type $x$-ray, which places the heaviest demand on the PACS.

The results presented are significant in that they show the suitability of the SAN formalism in modeling PACS. For the particular design considered, the results also provide useful information regarding times to perform system operations and use of resources. These performance figures are important in identifying system bottlenecks, and thus aid in the design of an efficient PACS. Specifically, we are able to (1) incorporate all the components of a PACS, ie, modalities, database, workstations, and network access protocols in a single model; (2) make use of the reduced base model formalism to represent the model in a modular manner; and (3) make use of the efficiencies of reducedbase model construction to reduce the time required to simulate the resulting model. ${ }^{6.7}$

In the remainder of the article, we first describe the PACS configuration and the operations of all the PACS components, namely, imaging modalities, the central database, viewing workstations and the local area network (LAN) connecting the different components together. We then briefly review the SAN and reduced base model construction formalisms and show their use in modeling a portion of an image database. Next, we provide detailed de- scriptions of the SAN models of all the components. Finally we discuss the simulation of the model and the performance figures obtained from the simulation. Performance values have been obtained with the aim of identifying system bottlenecks and predicting the response time of the PACS to image viewing requests under varying intensity and mixture of workloads.

\section{PACS MODEL DESCRIPTION AND ASSUMPTIONS}

In this section, we describe the PACS whose performance has been evaluated. The system consists of a central database, several diagnostic imaging modalities, and a number of image viewing workstations, as shown in Fig 1. The imaging modalities and the workstations are connected to the central database by a 100 megabits per second (Mbps) LAN.

We have chosen to model four X-ray machines (with digital input mechanisms), four ultrasound scanning machines, and one MRI unit. This PACS size is about the same as would be found in a 300 to 400 bed hospital in the United States. ${ }^{8}$ Table 1 provides data for the three modality types relevant to the modeling of the PACS, and was hypothesized using the American Hospital Association Hospital Technology Series $^{8}$ and statistics on available hardware. Machines of a particular modality type are identical in terms of hardware and imaging software. We have also assumed that there are a certain number of viewing workstations in the PACS and that all workstations have identical hardware and run the same system software. The number of viewing workstations is varied so

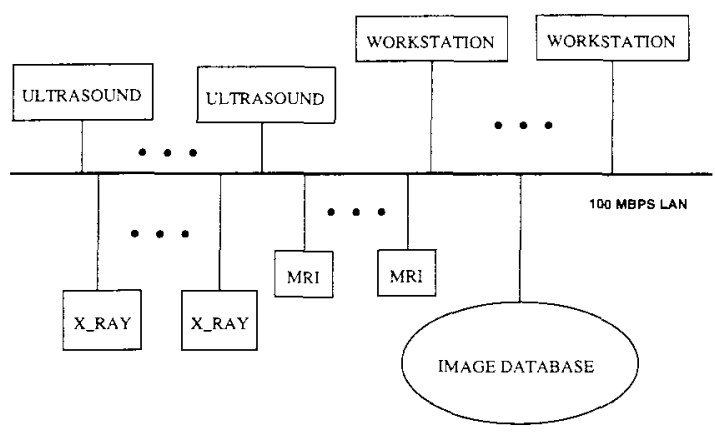

Fig 1. A picture archiving and communication system. 
that we may study the effect on system performance.

It must be emphasized that the configuration and design choices made in this section are to show the use of SANs in modeling PACS. Although they represent one possible configuration for a 300 to 400 bed hospital, many different configurations are possible and may lead to different performance results. The importance of this work is that it is possible to evaluate these alternatives for the workload and hardware configurations at a particular site.

\section{Imaging Modalities}

The main function of an imaging modality in a PACS is to generate diagnostic images of a patient and transmit these images over the LAN to the database. It is assumed that patients arrive at a modality at a fixed rate, with time between two patient arrivals being exponentially distributed. The number of images generated for a particular patient would, in general, be variable and would also depend on the modality type. Our model of an imaging modality is as follows. First, we assume that for each modality type, a fixed number of images is always generated, as shown in Table 1 . All the images generated for a patient are accumulated in a folder, and when the image generation process is complete, the folder is stored on a local hard disk. The newly generated folder is then queued up in a network interface buffer for transmission to the database. The delay for this procedure consists of the delay due to the writing of the folder to the hard disk, the time to read the folder from the hard disk and move it to the transmission queue, the queueing and transmission delay. These operations are shown graphically in Fig 2.

\section{Viewing Workstations}

Workstations are used in a PACS for viewing images of patients. These images may be newly generated images that have just been stored in

Table 1. Modality Data Relevant to PACS Modeling

\begin{tabular}{lccccc}
\hline \multicolumn{1}{c}{ Name } & $\begin{array}{c}\text { No. of } \\
\text { Units }\end{array}$ & $\begin{array}{c}\text { Arrivals/ } \\
\text { second }\end{array}$ & $\begin{array}{c}\text { No. Images } \\
\text { per Patient }\end{array}$ & $\begin{array}{c}\text { Image } \\
\text { Size (kb) }\end{array}$ & $\begin{array}{c}\text { Hard Disk } \\
\text { Speed (Mbps) }\end{array}$ \\
\hline MRI & 1 & 0.000175 & 32 & 98.3 & 2 \\
Ultrasound & 4 & 0.000075 & 36 & 0.786 & 2 \\
X-ray & 4 & 0.0012 & 3 & 5.24 & 2 \\
\hline
\end{tabular}

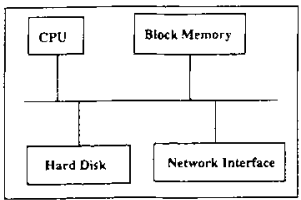

MODALITY HAROWARE

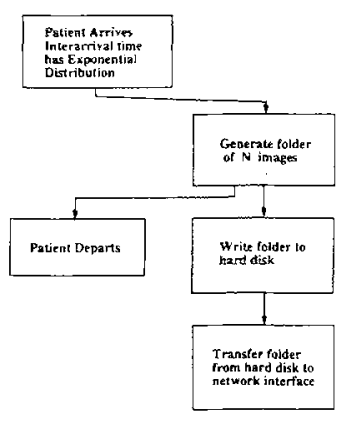

MODALITY OPERATION
Fig 2. Image generation at a modality.

the database, or images generated in the past and archived in the database. It is assumed that a radiologist is present at all workstations at all times during the day continually viewing sets of images.

A radiologist's work at a workstation is assumed to consist of a continuous cycle of requesting a set of images, waiting for these images to arrive at the workstation, and then viewing the set of images. Furthermore, we assume that a radiologist views an image set for an exponentially distributed length of time. We have varied this time to study its impact on the performance of the PACS. We have also varied the workload mix by retaining the modality type with the highest resource demand (x-ray) and turning off the other modalities to study its impact on the overall performance. We have assumed that a radiologist requests four images at a time. These images may have been generated at different modalities in the multiple modality case, or at the particular modality in the singlemodality case. The probability distribution of requests across modality types can be varied, and in the current study, is taken to be proportional to the normalized image generation rate (product of columns 3 and 4 ) at the various modality types as listed in Table 1 .

When the radiologist makes the request for an image set, the workstation operating system translates this request into four requests (for the four images) and queues them into a transmission buffer at the network interface hardware in the workstation. The requests are then transmitted serially to the database. The database examines each image request in turn, retrieves the image, and queues it in its transmis- 
sion buffer to send it to the requesting workstation.

An image received by a workstation is buffered in its network interface and then transferred on the data bus to the local block memory. From there, it is written to the local hard disk. The hard disk access rate in the workstation is assumed to be $2 \mathrm{Mbps}$. When the images have been received by the workstation and saved on its local hard disk, the request made by the radiologist is considered to be completely satisfied. The radiologist then views the image set for an exponentially distributed time before making the next request. Fig 3 shows the operation of a workstation as described above.

\section{Local Area Network}

The workstations and imaging modalities all share a common link to the database. This is an LAN with a capacity of $100 \mathrm{Mbps}$.

Imaging modalities use the network to transmit a folder of newly generated images to the

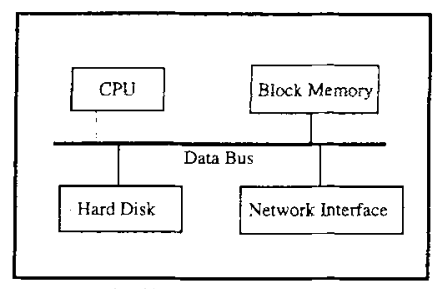

WORKSTATION HARDWARE
RADIOLOGIST WORK CYCLE

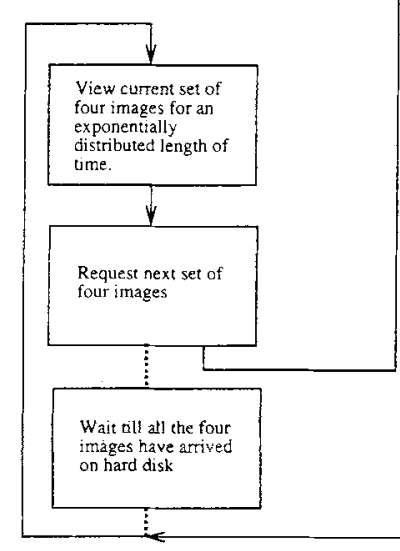

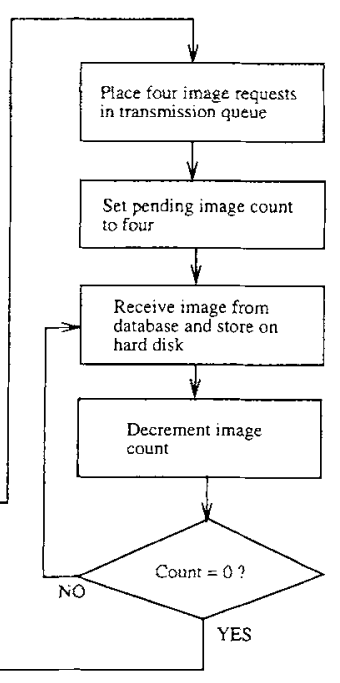

WORKSTATION OPERATION
Fig 3. Workstation operations. database for storage. The imaging unit queues up folders for transmission at the network interface buffer while awaiting the channel. This network interface buffer is common among all modalities and is capable of storing one complete folder generated by any given modality type. Transmission of a folder completes when the folder arrives at the database network interface buffer. The database at the other end cannot accept a new folder into its network interface buffer until the buffer has been cleared. This is done by removing a previously arrived folder from the buffer and sending it for further processing inside the database. After transmission is complete, the channel is released by the imaging unit and re-acquired if necessary.

Workstations use the network to transmit requests to the database and receive images from the database. As with imaging modalities, requests are transmitted one at a time, ie, the channel is released after every transmission and re-acquired if more requests are queued up in the transmit buffer. The workstation receives a requested image in its network interface buffer and can accommodate an image of the maximum size in the PACS. Again, the buffer has to be cleared of the previously received image to receive another image. As mentioned earlier, the database queues all incoming requests and processes each request one at a time. When the required image is retrieved, it is queued up for transmission and then transmitted one at a time to the requesting workstations.

These network operations are shown in Fig 4. Note that the media access control protocol is modeled very simply, without explicitly representing the control information that must be passed between nodes to achieve communication. This is because earlier studies ${ }^{9}$ have found that this is a negligible part of the total transfer time on the network, and hence negligible to the total response time of the PACS.

\section{Central Database Archive}

The central database archive is an integral part of the PACS. In addition to optical and hard disk storage, the hardware resources in the database include a network interface unit with finite buffer space and an image compressor unit. All these resources share a common data 


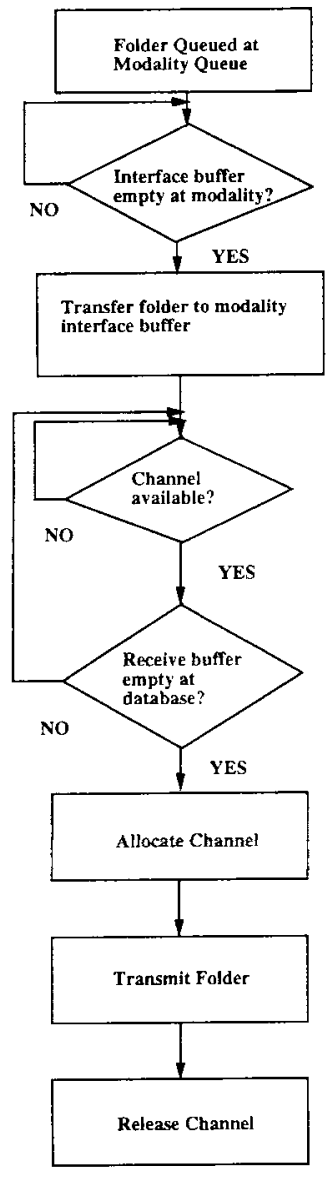

MODALITY TO DATABASE FOLDER TRANSMISSION

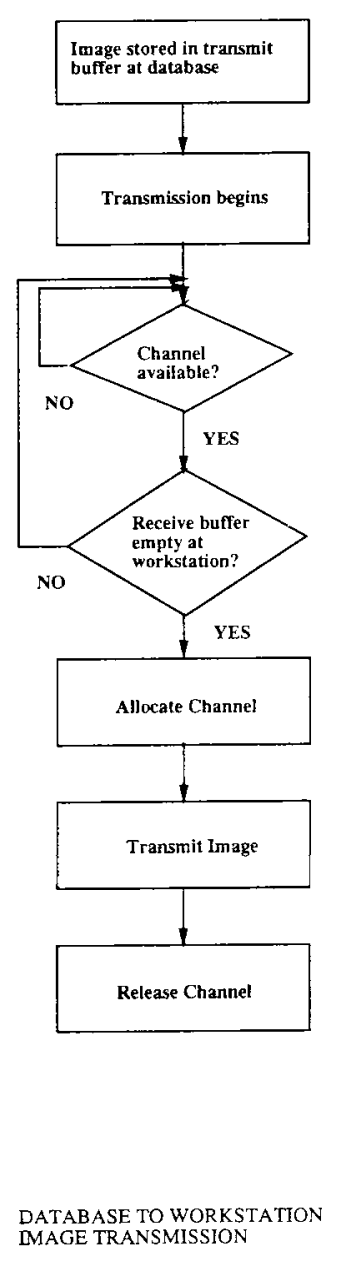

DATABASE TO WORKSTATTON

WORKSTATION TO DATABASE

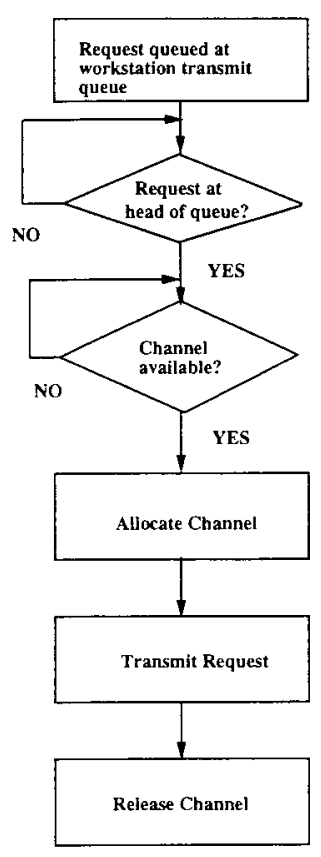
IMAGE REQUEST TRANSMISSION

Fig 4. Network operations.

bus inside the database system. Table 2 provides data relevant to modeling the database.

The database serves four main purposes: (1) Store newly generated images on the hard disk cluster in a compressed format; (2) Implement an algorithm for prefetching images from optical disk to hard disk; (3) Process image viewing requests from workstations; and (4) Maintain a long-term archive of images on the optical disk

Table 2. Database Data Relevant to PACS Modeling

\begin{tabular}{lc}
\hline \multicolumn{1}{c}{ Specification } & Value \\
\hline Compression Rate & 16M Pixels/s \\
Bits/Pixel: & 10 \\
X-ray & 24 \\
Ultrasound & 12 \\
MRI & $10 \mathrm{Mbps}$ \\
Hard disk assess rate & $300 \mathrm{kbps}$ \\
Optical Disk access rate &
\end{tabular}

by transferring all images on the hard disk cluster to the archive at the end of each working day.

The time required to read an image from the hard disk is much less than that required to perform a corresponding read from the optical disk. This in turn implies that the time to respond to a viewing request would be less if it were always located on the hard disk. But this may not always be the case because images generated in the past are archived on the slow optical disk storage unit. Hence, to increase the probability of locating a requested image on the hard disk cluster and thereby minimize the expected response time, a prefetching algorithm is modeled. In this algorithm, a write to the hard disk due to an incoming folder generates (with a certain probability) a request to read a folder that already exists on the optical 
archive. The interpretation is that a related folder is prefetched to the hard disk, eg, a past folder of the same patient. We note in this context that a write to the hard disk could occur either due to a newly generated incoming image folder, or to fetching a particular image that was requested by a viewing workstation from the stored optical archive if it was not already available on the hard disk. Because a prefetch is performed only on receipt of a new input folder, the algorithm first ascertains that this is indeed the case before proceeding with a prefetch.

The probability of prefetching a particular image folder is dependent on the image generation rate at the various modality units. From the data in Table 1 , it is observed that this rate (product of columns 3 and 4 ) is a maximum for the MRI unit and a minimum for the ultrasound unit, with that for the $x$-ray unit falling in between. Thus, the probability of a prefetch (given by the normalized image generation rate) is the highest for the MRI folder and the least for the ultrasound folder, with that for the $\mathrm{x}$-ray folder falling in between. Second prefetches on a single incoming folder are prevented by incorporating appropriate checks in the database model. Finally, at the end of the working day, all the images present on the hard disk cluster are once again archived on the optical disk.

The database operating system schedules a set of seven image-related hardware activities. The activities, which have been named in italics for convenience, are:

1. The transfer of a newly arrived image folder from the network interface receive buffer to the main memory area (niu_ bm-xfer).

2. The passing of an uncompressed (newly arrived) image folder through the image compression unit and the writing of the compressed data back into main memory (compression).

3. The writing of a compressed image folder to the fast hard disk cluster in the database (bm_hd_xfer).

4. The reading of an image or folder from the slow optical disk archive (od_read).

5. The searching of the image database cata$\log$ to locate the requested image (catalog-search).
6. The reading of a compressed image from the hard disk cluster and its subsequent write to the main memory ( $h d_{\text {_read) }}$.

7. The transfer of a compressed image from the main memory to the network interface transmit buffer $\left(b m_{-} n i u_{-} x f e r\right)$.

Figure 5 is a flowchart of activity sequences for image storage, prefetching, and retrieval in the database. The scheduling algorithm we model divides the above activity set into two subsets. Subset A contains activities bm_niu_ $x f e r, h d_{\text {read, }}$ and catalog_search; these are associated with the processing of requests generated by the workstations. Subset B contains activities od_read, bm_hd_xfer, compression, and niu_bm $x f e r$, which are in turn connected with new folder acquisition and storage and a corresponding prefetch. The central processing unit (CPU) of the database is required for execution of each activity. A CPU allocation request for an activity in this set is usually made by another activity in the same set. Subset $A$ has a higher scheduling priority than subset $\mathrm{B}$. Also, within a subset, the activities have been listed above in the order of their priority, eg, activity od_read has the highest priority in subset $\mathrm{B}$. This is just one possible scheduling algorithm, others could also be studied.

There is a certain amount of coupling between the two subsets, as is now explained. Based on the location of the requested image, completion of catalog_search is followed by either one of the following two sets of activity sequences. If the image is located on the hard disk (a hit), then activity hd_read is scheduled which is followed by a bm_niu_xfer, both of which belong to subset A. If, on the other hand, the requested image is located on the optical disk (a miss), it must first be read off of the optical disk before it can be transferred to the hard disk to be dispatched to the workstation. This now implies a scheduling of the activity sequence od_read and $b m_{-} h d_{-} x f e r$, both of which belong to Subset $\mathrm{B}$, before scheduling hd_read and $b m_{-} n i u_{-} x f e r$. Thus, based on the probability of a miss, we observe that a certain amount of coupling exists between the two subsets of activities. However, for lower response times, the probability of this coupled sequence should be very low, as it corresponds to the probability 


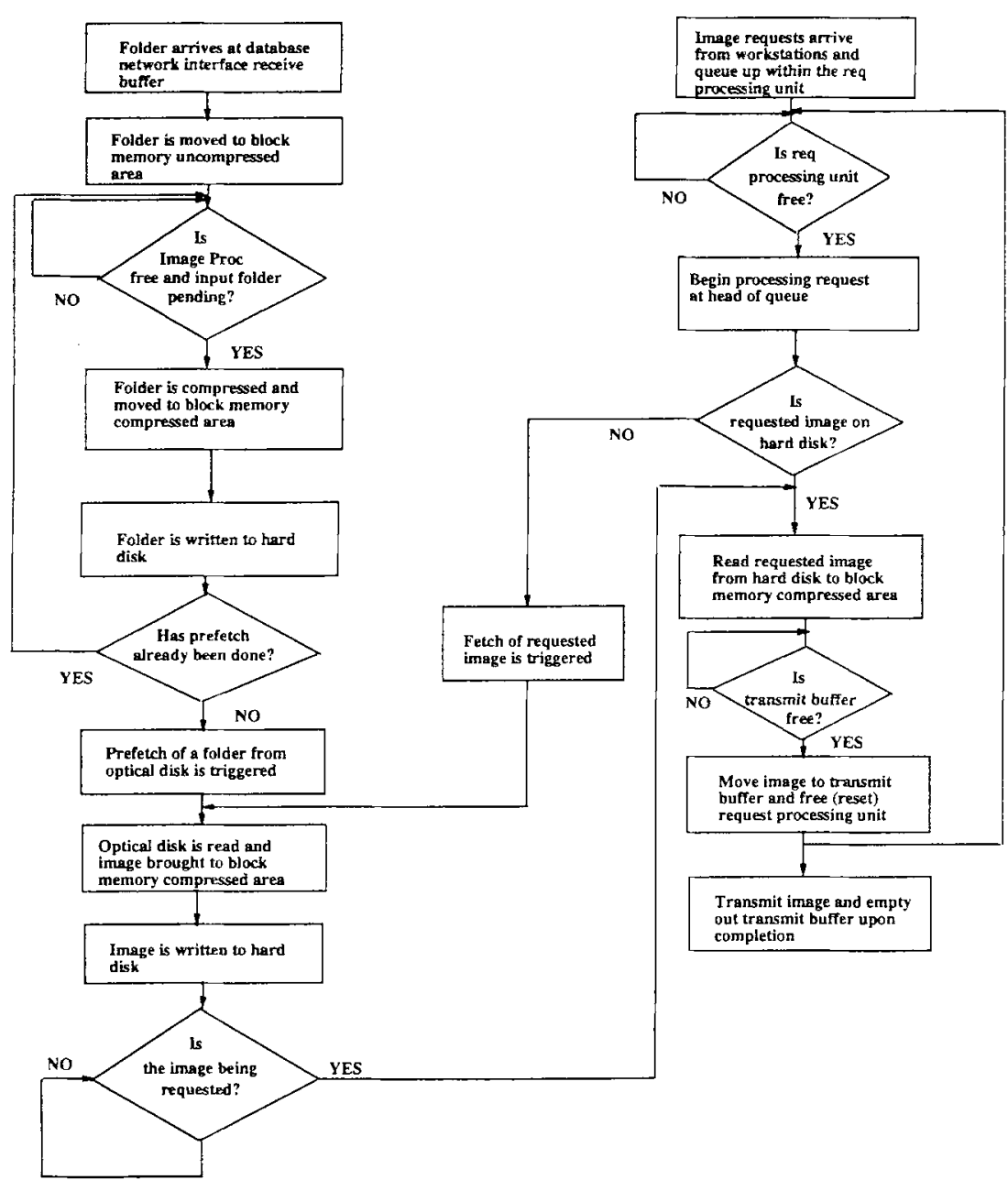

IMAGE STORAGE AND PREFETCH
VIEWING REQUEST PROCESSING
Fig 5. Database operations. of the image not being present on the hard disk for fast reading.

The PACS under consideration is built from the four component types connected together via the LAN. In the next section we provide a brief review of the SAN modeling formalism and then describe the modeling of a PACS configuration using SANs.

\section{MODELING FRAMEWORK}

The models used are hierarchical, with stochastic activity networks of individual components replicated and joined together with other models.

\section{SANS}

SANs are a stochastic extension to Petri nets. ${ }^{3.4}$ Structurally, they consist of activities, places, input gates, and output gates. To illustrate activities and other SAN components, we consider an SAN model of a part of a PACS database, shown in Fig 6. The function of this model will be explained later; we introduce this model here only to illustrate the components that make up an SAN model.

Places are used to represent the "state" of a system (eg, places im_niu_mri, bm_out_place, od_read_place, etcetera, in Fig 6) and may contain tokens. The number of tokens present in a place is called the marking of that place. The marking of the SAN is the vector consisting of the markings of all the places in the SAN. Tokens in a place may be given different interpretations, depending on the purpose of the particular place in the model. For example, the number of tokens in bm_out_place represents 


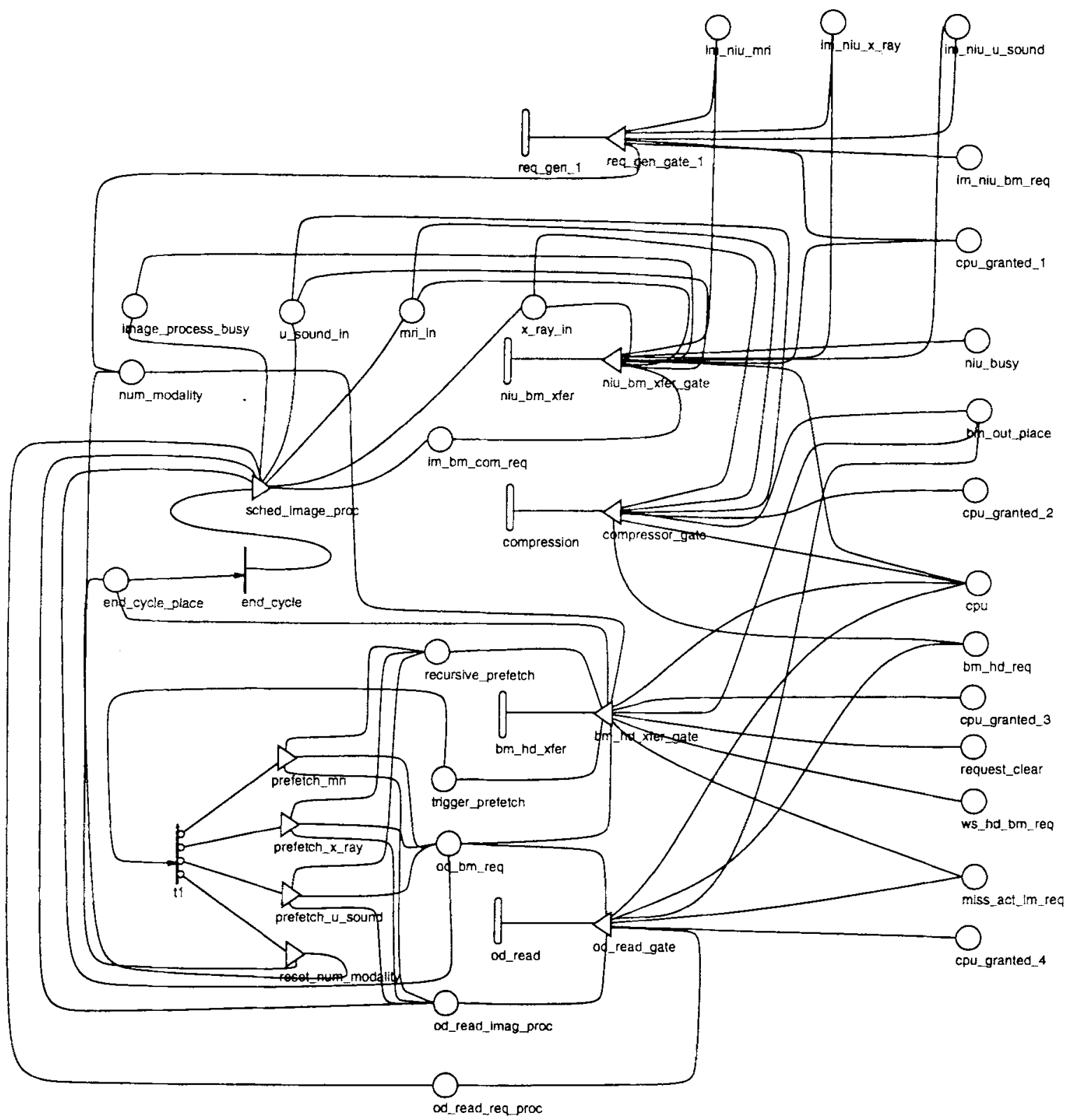

Fig 6. SAN representation of image acquisition and storage operations.

the number of bytes of image data in the block memory of the database, while that in cpu indicates the availability or nonavailability of the CPU.

Activities, which are similar to transitions in normal Petri nets, are of two types: timed and instantaneous. Timed activities ( $\mathrm{eg}, b m_{-} h d_{-} \times f e r$ ) represent activities of the modeled system whose durations impact the system's ability to perform. Instantaneous activities (eg, $t 1)$ on the other hand, represent system activities which, relative to the performance variables in question, complete in a negligible amount of time. When an activity completes, one token is removed from each of the places directly connected (ie, not through a gate) to the input of the activity, and one token added to each of the places directly connected to the output of the activity. Table 3 shows the timings associated with the activities present in Fig 6. As can be 
Table 3. Activity Timings for Image Processing

\begin{tabular}{|c|c|}
\hline Activity & Distribution \\
\hline$b m_{-} h d_{-} x f e r$ & deterministic(MARK(bm_out_place) $* 0.001$ ) \\
\hline \multirow[t]{7}{*}{ compression } & if((MARK(mri_in)) \\
\hline & return(deterministic(MARK(mri_in) *0.0004166)) \\
\hline & else \\
\hline & if(MARK(x_ray_in)) \\
\hline & return(deterministic $\left(\right.$ MARK $\left(x\right.$ ray_in) $\left.\left.{ }^{*} 0.0005\right)\right)$ \\
\hline & else \\
\hline & return(deterministic(MARK(u_sound_in) * 0.00020833 )) \\
\hline \multirow[t]{2}{*}{ niu_bm_x fer } & deterministic((MARK(im_niu_x_ray) + MARK(im_niu_u_sound) \\
\hline & + MARK(im_niu_mri) $\left.)^{*} 0.0005\right)$ \\
\hline \multirow[t]{4}{*}{ od_read } & if(MARK(od_read_req_proc)) \\
\hline & return(deterministic(MARK(od_read_req_proc) $\left.{ }^{*} 0.0333333\right)$ ) \\
\hline & else \\
\hline & return(deterministic(MARK(od_read_imag_proc) $* 0.033333)$ ) \\
\hline req-gen_t & deterministic $(0.00000000000001)$ \\
\hline
\end{tabular}

seen, the activity timing can depend on the marking of places in the network. For example, activity $b m_{-} h d_{-} x f e r$ has a deterministic timing which is dependent on the marking of place bm_out_place. Although all the timed activities in this submodel have deterministic times associated with them, activity times can be generally distributed.

Cases associated with activities (represented as small circles on one side of an activity) permit the realization of uncertainty concerning what happens when an activity completes. In our example SAN, activity $t l$ has four cases. The choice of a particular case decides the size of a folder to be retrieved (prefetched) from the optical disk in the database. As with activity times, case probabilities can be dependent on place markings. Table 4 gives the case probabilities associated with activity $t$.

Input gates and output gates permit greater flexibility in defining enabling and completion rules than with regular Petri nets. In particular, input gates have enabling predicates and functions, while output gates have only functions. The enabling predicate can be any computable predicate (taking on true and false values) of the places connected to it, and, as seen in that which follows, controls the enabling of an at-

Table 4. Activity Case Probabilities in Image Processing

\begin{tabular}{lcl}
\hline Activity & Case & Probability \\
\hline$t 1$ & 1 & 0.2352941 \\
& 2 & 0.1512605 \\
& 3 & 0.11344531 \\
& 4 & 0.5
\end{tabular}

tached activity. The function associated with each input or output gate describes an action (change in marking) that will occur on completion of the activity. As with predicates, gate functions can be any computable function on the places connected to the gate.

Activities are enabled if there is at least one token in each of the places directly connected to the activity and if the predicate of each connected input gate is true (ie, holds). As an example, consider activity compression which has input gate compressor_gate connected to it. This activity models the compression of an image folder to half its original size. According to the activity enabling predicate specified in Table 5, the activity is enabled only when the CPU has been allocated for compression work. If the predicate remains true, then the activity will complete, after a time determined by its activity time distribution. On completion, the gate function, as specified in Table 5, will be executed causing tokens to be removed from certain places and/or transferred to other places.

Output gates, together with directly connected output places, are used to specify the action to be taken on completion of an activity. The function associated with each output gate can be any computable function on the connected places. For example, as shown in Table 6 , output gate od_bytes 1 deposits 786 tokens in place od_read_place, corresponding to a compressed x-ray folder of size approximately 7.86 megabytes, and places a request token in od bm_req to schedule a read operation on the optical disk. 
Table 5. Input gate specification for image processing

\begin{tabular}{|c|c|c|}
\hline Gate & Enabling Predicate & Function \\
\hline compressor_gate & MARK $($ cpu_granted_3 $)==1$ & 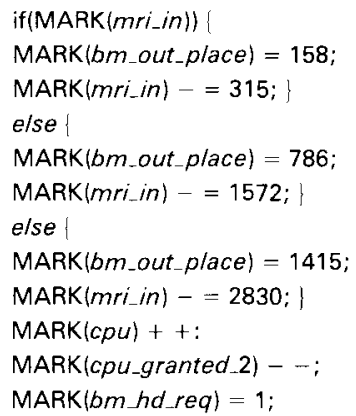 \\
\hline
\end{tabular}

Given the brief description of stochastic activity networks above, it is possible to construct models of small systems that permit solution for a specified set of performance variables. For large-scale systems, a model construction technique known as "reduced-base model construction"s greatly enhances the suitability of the SAN formalism for modeling systems with many different physical and logical entities with multiple copies of each entity possibly present in the system. This construction technique is highly modular in nature and takes advantage of the symmetries in the overall SAN structure. The reduced base model construction technique has been incorporated into UltraSAN ${ }^{6,7}$ and has been used in the modeling of the PACS described in this article.

To use this approach, a complete (or composed) model is built from one or more SAN submodels using replicate and join operations. The replicate operation replicates a submodel a certain number of times. Thus the replicate operation allows one to construct composed models that consist of several identical component submodels. The combination of several different submodels is accomplished using the

Table 6. Output Gate Functions for Image Processing

\begin{tabular}{|c|c|}
\hline Gate & Function \\
\hline pre fetch_mri & $\begin{array}{l}\text { MARK }(\text { recursive } p \text { pre fetch })=1 \\
\text { MARK }\left(o d_{-} \text {read_image_proc }\right)+=158 \\
\text { MARK }\left(o d_{-} b m_{-} r e q\right)=1\end{array}$ \\
\hline pre fetch_x_ray & $\begin{array}{l}\text { MARK }(\text { recursive_prefetch })=1 \\
\text { MARK }(\text { od_read_image_proc })+=786 \\
\text { MARK }(\text { od_bom_req })=1\end{array}$ \\
\hline pre fetch_u_sound & $\begin{array}{l}\text { MARK (recursive_prefetch })=1 ; \\
\text { MARK }(\text { od_read_image_proc })+=1415 ; \\
\text { MARK }\left(o d_{-} b m_{-} \text {req }\right)=1 ;\end{array}$ \\
\hline
\end{tabular}

join operation. The join operation produces a composed model which is a combination of the individual submodels. An example of the use of these operations will be given in the next section, in which a complete model of the PACS is also given.

\section{PACS MODEL DESCRIPTION WITH SANS}

The PACS under consideration has been modeled using composed SAN models. Modeling with composed models and SANs allows the model to be decomposed into smaller submodels of logical entities, such as a scheduling algorithm for the database CPU, or physical components, such as an imaging modality. There are several identical units in the system (eg, $x$-ray machines), in a PACS, and these can be represented by one SAN submodel replicated as many times as there are units, using the replicate construction operation described earlier. All the SAN submodels of the system are joined together in a hierarchical fashion, using the join operation at appropriate stages in the model building process.

Figure 7 shows the composition of the PACS model using the model construction techniques described earlier. The system model has been divided into three main parts: the imaging modalities, the database and the workstations. There are three different imaging modalities in our model, namely, x-ray, ultrasound, and MRI.

SAN submodels for each of these modality types are structurally very similar except that submodel parameters are different for each type. The modality submodel is further split up into the modality operational submodel and the modality network interface submodel. These 


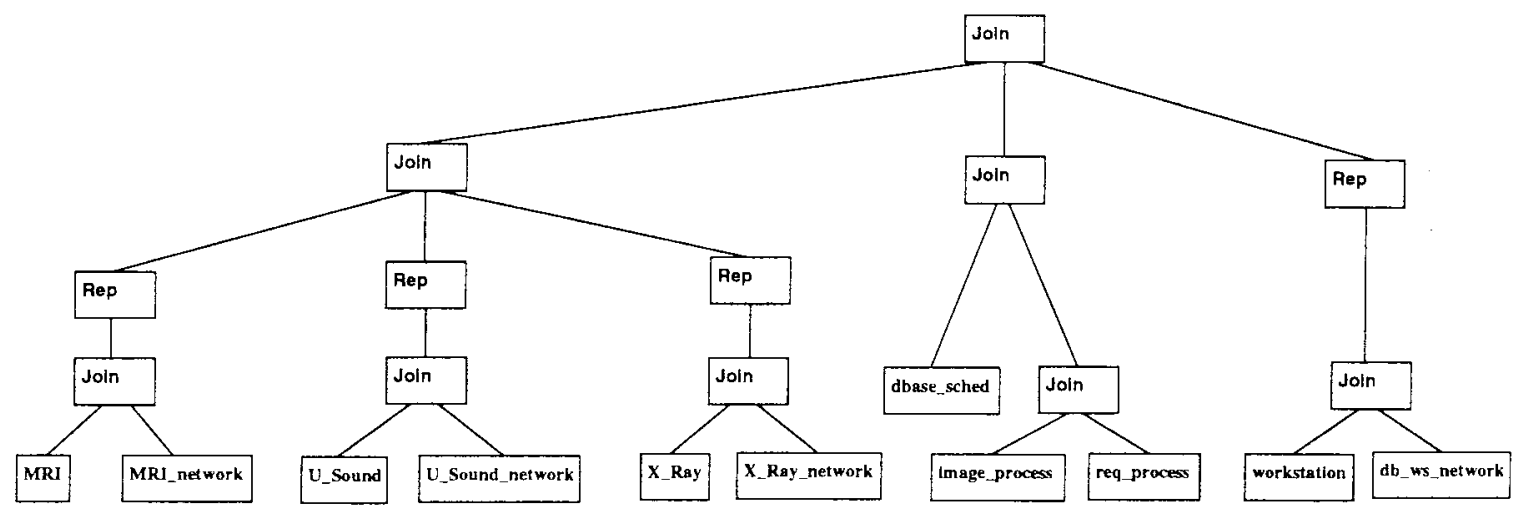

Fig 7. Composed SAN model of PACS of Fig 1.

two submodels are joined to form the modality submodel which is then replicated to represent several units of that modality type. Replicated submodels of different imaging modalities are then joined together to form a common node which connects to the database and workstation submodels using another join at the topmost level in the model hierarchy. The workstation submodel is similarly constructed, building upward from the workstation operational SAN submodel and the workstation network interface SAN submodels. It is indeed this modular nature that has enabled us to reconfigure the PACS with a great deal of ease and thereby investigate its performance under different system configurations.

The database submodel has been split into three SAN submodels: the image acquisition submodel, the viewing request processing submodel, and the CPU scheduler submodel. The image acquisition submodel represents the activities in the database related to new image acquisition and storage, and the image prefetching algorithm. The viewing request processing submodel describes database activities connected with the processing of image viewing requests arriving from viewing workstations. These submodels are joined together and again joined with the scheduler submodel. All the submodels are linked together by a common join which is the topmost node in the composed model.

In the following subsections, the SAN models of the different PACS components are briefly explained. Important input/output gate specifications, activity time distributions, and case probabilities for each of the submodels described below have been included in. ${ }^{10}$

\section{Imaging Modality Submodel}

The SAN model of an imaging unit consists of two submodels that are then joined together. They are an operational submodel and a network interface submodel. Figure 8 is the SAN representation of the operational model. Activity cust_arr models the arrivals of imaging jobs (patients) at a modality, whereas place cust queue represents the queue of these jobs at the corresponding modality. A job corresponds to the generation of a folder of images, with the number of images per folder (and hence per patient), depending on the modality type. For example, from Table 1, the number of images generated per patient for an MRI unit is 32 while those for ultrasound and x-ray units are 36 and 3 , respectively. On generation, the image folders are first written to the hard disk and

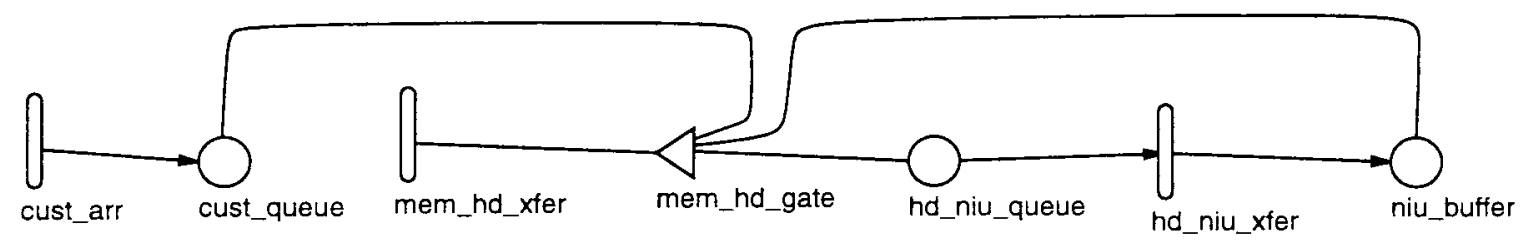

Fig 8. SAN representation of modality operation. 
then transferred across the network to the central database archive.

There are two image folder data transfers which use the CPU of a modality: the transfer from the modality random access memory (RAM) to its hard disk (activity mem_hd $x$ fer and place $h d_{-}$niu_queue), and the transfer from hard disk to the network interface memory (activity $h d_{-} n i u_{-} x f e r$ and place niu_buffer). The model ensures (by using predicates to inhibit activities) that two activities that use the CPU cannot take place at the same time. The CPU is thus not modeled explicitly as a resource in this subnet.

The network interface buffer is represented by niu_buffer and is common to all modalities. A new folder cannot be written to this buffer until the previously written folder has been transmitted across the network and deposited at the corresponding buffer on the database side. This is again taken care of by the use of appropriate gate predicates in the SAN model. Finally, the image folder transfer times depend both on the modality hard disk speed and on the size of the particular folder. The former is assumed to be 2 MBps whereas the latter is obtained by multiplying columns 4 and 5 of Table 1 .

Figure 9 is the SAN representation of the network interface connecting a modality to the database. Place niu_buffer is the modality network interface buffer discussed earlier. The network interface buffer at the database side of the channel is represented by im_niu_modality and is common to all modalities of a given type. Place niu_busy is used to indicate the availability of the network interface buffer. The presence of a token in this place indicates that the network interface buffer is full. Thus, before attempting to obtain the channel, channel_grab_gate checks to see if this buffer is empty. The channel is acquired if this buffer is found to be empty, and if, of course, the channel itself is free. The availability of the channel is indicated by the presence of a token in place channel. The acquisition of the channel is accomplished by completion of activity channel_grab. The completion of channel_grab is accompanied by the following changes in the marking of this SAN. A token is removed from channel to indicate that it is busy. A token is added to each of the places niu_busy and xmit_place, with the former denoting the nonavailability of the network interface buffer and the latter representing the onset of transmission.

Once the channel has been allocated, transmission begins (activity xmit), and on completion of transmission, the image folder is written to the network interface buffer (place im_niu_ modality). The number of tokens placed in this buffer depends on the size of the image folder, which in turn is dependent on the modality type (Table 1). Because of the large sizes of various image types, a representational unit of $10 \mathrm{~kb}$ for every one token is used in the model. Thus, for example, 315 tokens are used to represent an uncompressed MRI folder of size $3145.6 \mathrm{~kb}$. As before, the transmission time for a folder depends on its size.

\section{Database Submodel}

The database submodel consists of the SAN representations of the database CPU scheduler,
Fig 9. SAN representation of modality network interface.

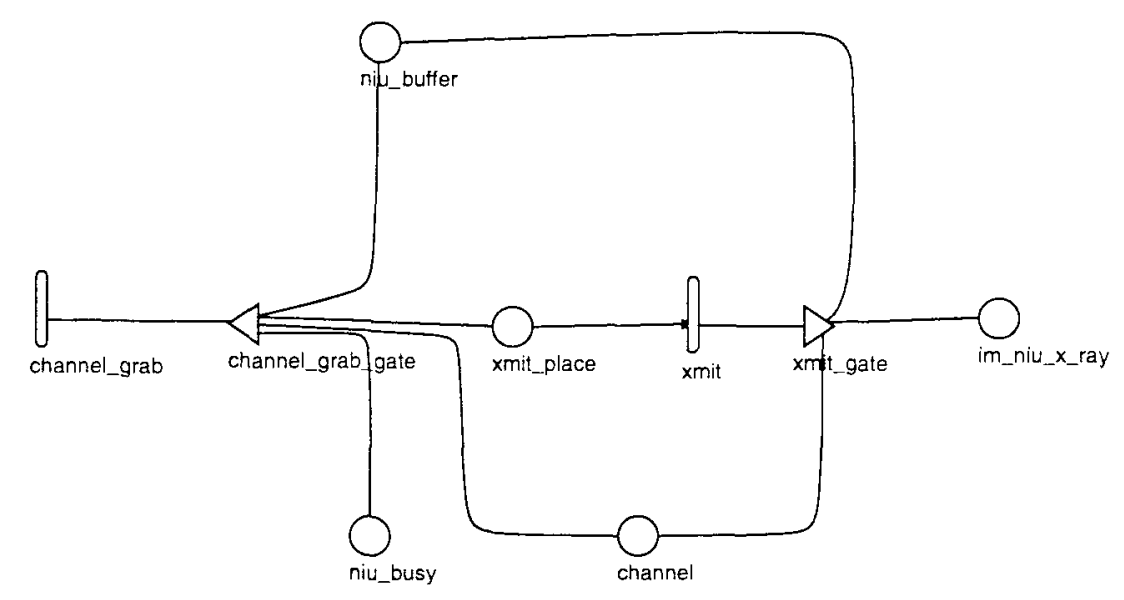


the image acquisition and storage submodel, and the viewing request processing submodel. The image acquisition and storage submodel was shown earlier in Fig 6. The scheduler and viewing request processing submodels are shown in Figs 10 and 11 , respectively. These SAN representations are joined together, as shown earlier in Fig 7.

The scheduler SAN consists of two activities, high act and scheduler, with the former having a higher priority than the latter. Tokens in each of the places located to the left of these activities (ie, places ws_req through od_bm_req) represent CPU requests while those in corresponding places to the right (places cpu_granted_l through cpu_granted_7) denote the granting of the CPU for the required operation. The scheduling of activities in subset $\mathrm{A}$ is controlled by input gate high_sched while that in subset $\mathrm{B}$ is performed by scheduler. A request for the CPU in any of the above mentioned request places is first checked for by the appropriate input gate and, based on the priority rules, the CPU is granted by removing a token from place $c p u$ and placing it in the appropriate $c p u_{-}$granted_number place. On completion of the requested activity (which may be either in subset $A$ or subset $B$ ), a token is removed from the $c p u_{-}$granted_number place and returned to the place $c p u$.

The image acquisition and storage SAN (Fig 6 ) is used for acquisition and storage of newly generated image folders, prefetching of subsets of image folders from the stored archive of images, and fetching of requested images that are stored on the optical disk but are not present on the hard disk. The im_niu_modality places represent the database network interface buffer. Recall that this buffer space is common to all modalities, and we can at any given instant, have only one image folder residing in this buffer space. This is taken care of by placing a token in niu_busy on the arrival of a folder to any of the im_niu_modality places. Because niu_busy is common to all modalities, it is ensured that the network interface buffer will never overflow.

The arrival of an image folder is sensed by input gate req-gen_1, which then proceeds to request the CPU by placing a token in im_niu. $b m_{-}$req. Places modality_in represent the queue of jobs (image folders) within the central database that are waiting to be compressed and archived. The time required for this transfer (time for activity im_niu_xfer), is dependent on

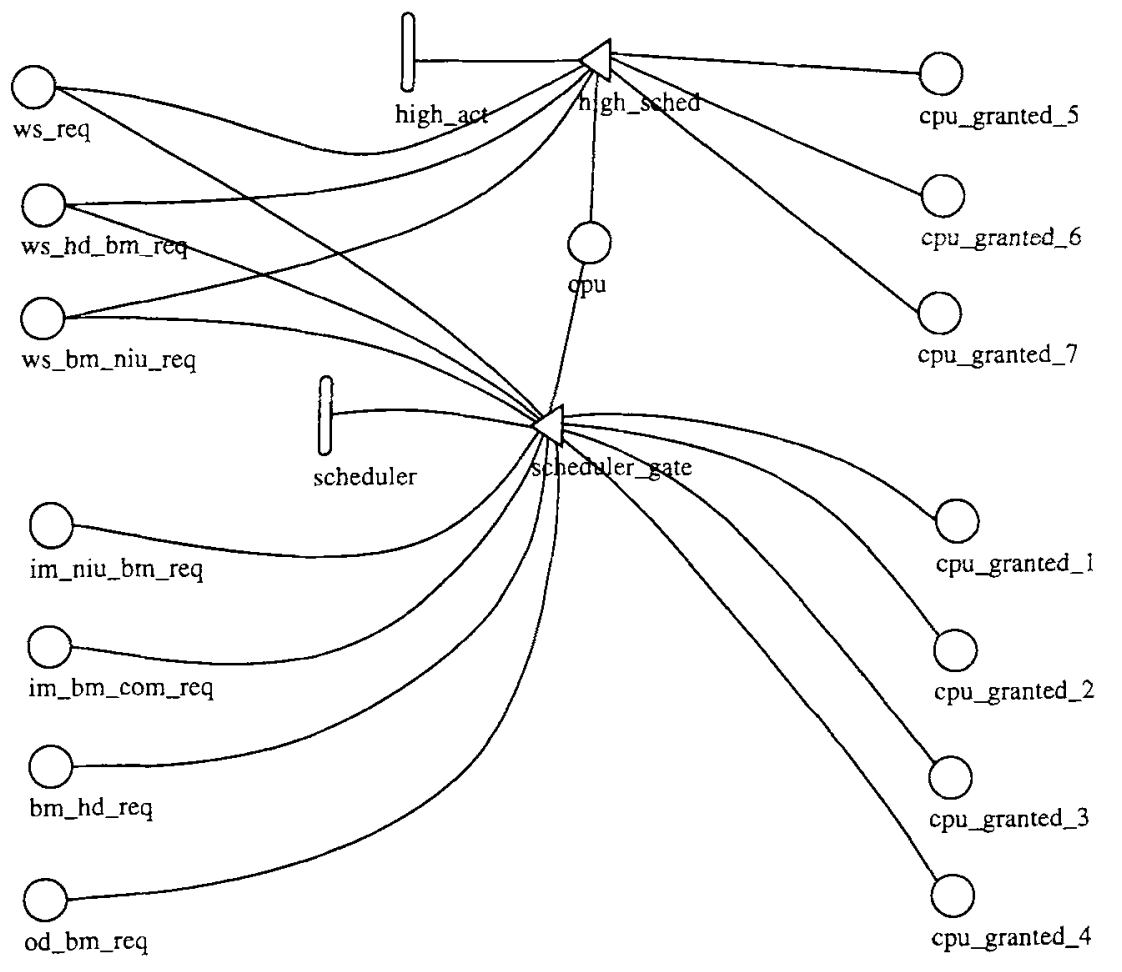

Fig 10. SAN representation of database scheduling algorithm. 
Fig 11. SAN representation of viewing request processing operations.

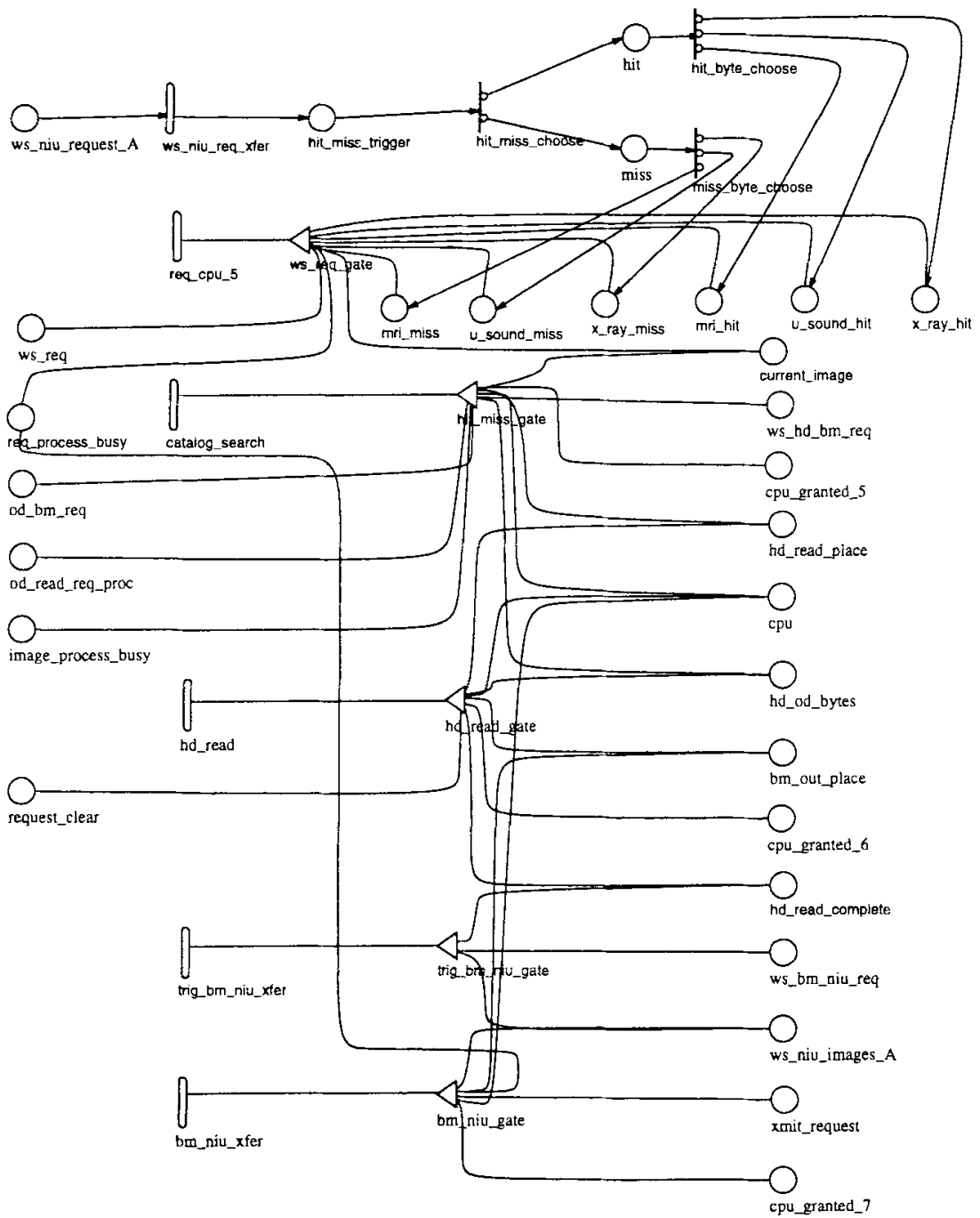

the image size. The hard disk speed for the database is assumed to be $10 \mathrm{Mbps}$.

Completion of activity im_niu_xfer is accompanied by the following actions. First, the contents of the database network interface buffer are transferred to the appropriate modality_in place and the CPU is released. This is followed by clearing the niu_busy place to indicate the fact that the interface buffer has now been emptied. Finally, the image processing unit is checked to see if it is free by checking the number of tokens in place image_process_busy, which contains one token if any of the activities compression, $\mathrm{bm}_{-}$ $h d_{-} x f e r$, or od_read is busy, and zero otherwise. If it is ascertained to be free, a CPU request to initiate the image compression phase is scheduled, and a token is placed in image process busy to indicate that the image processing subunit is now busy.

The time required to compress an image folder depends on the image pixel size and the compression rate. These values are obtained from Tables 1 and 2. In the case of multiple folder types, the modality_in places are scanned in the order of their folder size and the first non-empty image folder is chosen for compression. On completion of compression, the folder data is compressed to half its original size and moved on to bm-out_place and a request is then made for the next activity in sequence, $b m$. $h d_{-} x f e r$.

Activity $b m_{-} h d_{-} x f e r$ is used to move the compressed folder to the hard disk. Completion of this transfer triggers the image-folder prefetch 
algorithm which functions in the following manner. First, the input data size is checked to ascertain whether it is an image folder or just a single image. If it is an image folder, the algorithm proceeds to check if this is the first prefetch to avoid a second prefetch on a single folder. Second prefetches are indicated by the presence of a token in the place recursive_prefetch. If this happens to be the first prefetch, then activity $t 1$ is enabled, which performs a prefetch with a $50 \%$ probability. If, on the other hand, a prefetch has been performed, then no more prefetching is done for the current folder, and the cycle is ended by triggering activity end_cycle.

The probability of prefetching a particular image type, as discussed above (see Central Database Archive section) is proportional to its generation rate. Hence, for the present PACS, these probabilities for the MRI, x-ray, and ultrasound folders assume the case values listed in entries 1 through 3 , respectively, of Table 4 . The fourth case corresponds to the probability that no image-folder prefetch is triggered. Finally, as the folder sizes differ for the different modality types, the output gates prefetch_mri, prefetch_o_ray, and prefetch_u_sound are used to place different numbers of tokens in place od_read_imag_proc, and a CPU request token in od_bm_req.

Image requests generated by the workstation units are processed by the viewing request processing submodel that is shown in Fig 11. The arriving image request tokens are first stored in ws_niu_request, and, after a short deterministic delay (ws_niu_req_xfer), are transferred to place hit_miss_trigger. This delay represents the time to transfer a finite size request token from the network interface buffer (ws niu_request) to the buffer space within the database. The size of a request token is taken to be 100 bytes. Activity hit_miss choose is used to determine if the image corresponding to the current request is located on the hard disk (a hit, .99 probability) (Fig 20) or on the optical disk (a miss, .01 probability). The size and hence type of the requested image in both cases (hit or miss) is determined from the case probabilities on their respective activities (hit_byte_ choose or miss_byte_choose). The distribution of these case probabilities is, as mentioned before, proportional to the image generation rate (refer to columns 3 and 4 in Table 1) and is included in Table 20.

Places modality_hit and modality_miss represent the queue of jobs (image viewing requests) within this submodel because all arriving requests are translated into either a hit or a miss and are stored in these modality hit/miss queues. On arrival of a particular image request, the following functions are performed by the input gate ws_req_gate. First, the request processing submodel is checked to see if it is free, ie, if none of the activities catalog_search, hd_read, triq$b m_{-} n i u_{-} x f e r$, and bm_niu_xfer are busy. This is done by ascertaining that place req_process_busy has no token in it. (A token in req_process_busy indicates that this submodel is busy.) If it is found to be free, a token is placed in ws_req to request that the CPU perform the catalog search. Next, the modality_hit and modality_miss places are scanned in an increasing order of their image size, and a certain number of tokens corresponding to the first non-empty image request is placed in current_image. Finally, a token is placed in req_process_busy to indicate that this unit is now busy processing a request.

After the completion of catalog search (activity catalog_search with a time of 1 millisecond), the following set of actions are performed. If a hit has occurred, then the image may be fetched from the hard disk, which results in a scheduling request for activity $h d_{-}$read. If, on the other hand, the image was not located on the hard disk cluster, a miss has occurred. The requested image must therefore be fetched from the optical disk. This is indicated by placing a certain number of tokens (corresponding to the size of the desired image whose type is stored in current_image) in od_read_req_proc and scheduling a CPU request for an optical disk read. However, because optical disk reads are performed by the image processing unit and as that unit could be busy, place image_process_busy is first checked to see if it contains a token. If it does not (which implies that the image processing subunit is currently free), a scheduling request for activity od_read is made by placing a token in $o d_{-} b m_{-} r e q$. If, on the other hand, the image processing subunit is found to be busy, no immediate request for the CPU is made. In this case, place od_read_req_proc contains tokens 
that also serve as an indicator to the image processing unit to process this request as soon as it is done processing its current job.

From the discussion above, we observe that activity $h d_{-}$read may be triggered under two circumstances, namely, during a hit or a miss. In the former case, however, it could be made to complete with a smaller delay (a delay corresponding to the hard disk read of the corresponding image), while in the latter case it would need to wait until the optical disk read is complete (indicated by the presence of a token in request_clear) and the image is fetched and placed into hd_od_bytes. The sequence of actions after this is common to both the hit and miss cases. A token is placed in hd_read_complete to indicate the completion of a hard disk read. Activity trig_bm_niu $x f e r$ is used to admit one image at a time to the transmission buffer ws_nili_images. It is also used to place a $\mathrm{CPU}$ request to transfer the image from the database buffer memory to the transmission buffer. The time to accomplish the above mentioned transfer (database buffer memory to transmission buffer) is represented by bm_niu_xfer and is dependent on the size of the requested image (see Fig 19).

\section{Workstation Submodel}

The SAN representation of a workstation is shown in Fig 12. Activity viewing-time, as its

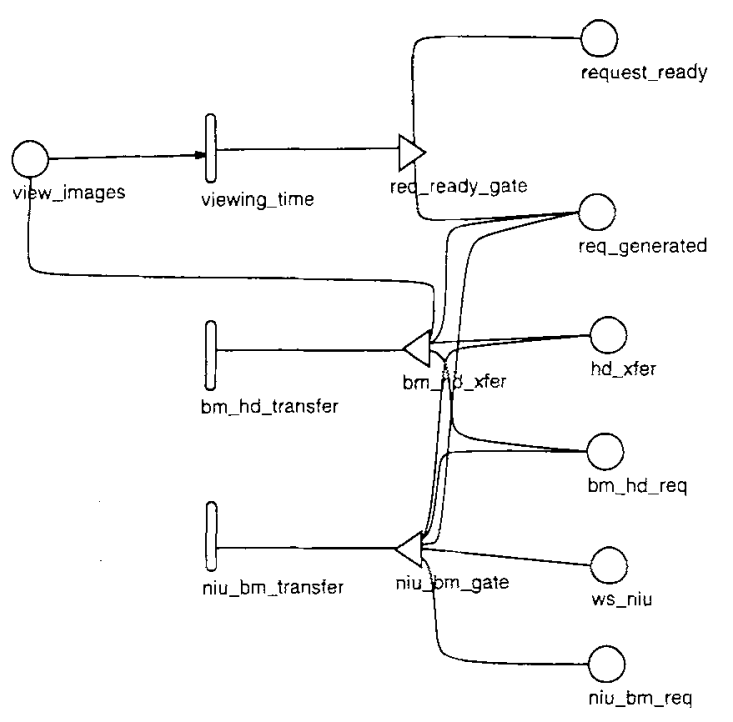

Fig 12. SAN representation of workstation operation. name denotes, represents the time taken to view the set of requested images. An exponential viewing time and a set of four images per request set have been assumed. Output gate req_ready_gate is executed on completion of activity viewing_time and deposits four tokens in each of the places request_ready and req_generated. Activity niu_bm_transfer represents the time required to transfer the requested image from the transmission buffer to the block memory in the workstation, while bm_hd_transfer denotes the time to copy this image on to the hard disk. Because both these activities need to use the CPU of the workstation, the model ensures, via the use of indicator places niu $b m_{-} r e q$ and $b m_{-} h d_{-} r e q$, that these two activities refrain from using the CPU simultaneously. The transfer times (niu_bm_transfer and bm_hd_transfer) are marking dependent on the image size and a workstation hard disk speed of 2 Mbps has been assumed. The number of tokens in req_generated represent the number of outstanding images within the current image set, and is decremented by one (by niu_bm_gate) each time an image is sent to the corresponding workstation. Finally, after checking to see if all the four images in the given image set have been re-

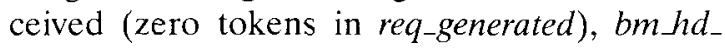
$x f e r$ deposits a token back in place view_images.

The SAN representation of the network interface between a workstation and a database is shown in Fig 13. The top half of this figure represents the set of places and activities involved in the transmission of an image request token from the workstation to the database, while the bottom half is used to represent the places and activities instrumental in transmitting the retrieved image back to the workstation. The workstation deposits its image request token in request_ready, which is then transmitted across the channel to the request processing unit of the central database. The image, on retrieval from the database, is stored in the transmission queue buffer place $w s_{-} n i u_{-}$images. The channel access protocol is the same as that used for transmitting image folders to the database from the modalities. The transmission time $(x m i t)$ is dependent on the image size. Completion of image transmission is marked by transferring the contents of ws_niu_images over to the workstation interface buffer ws_niu. 


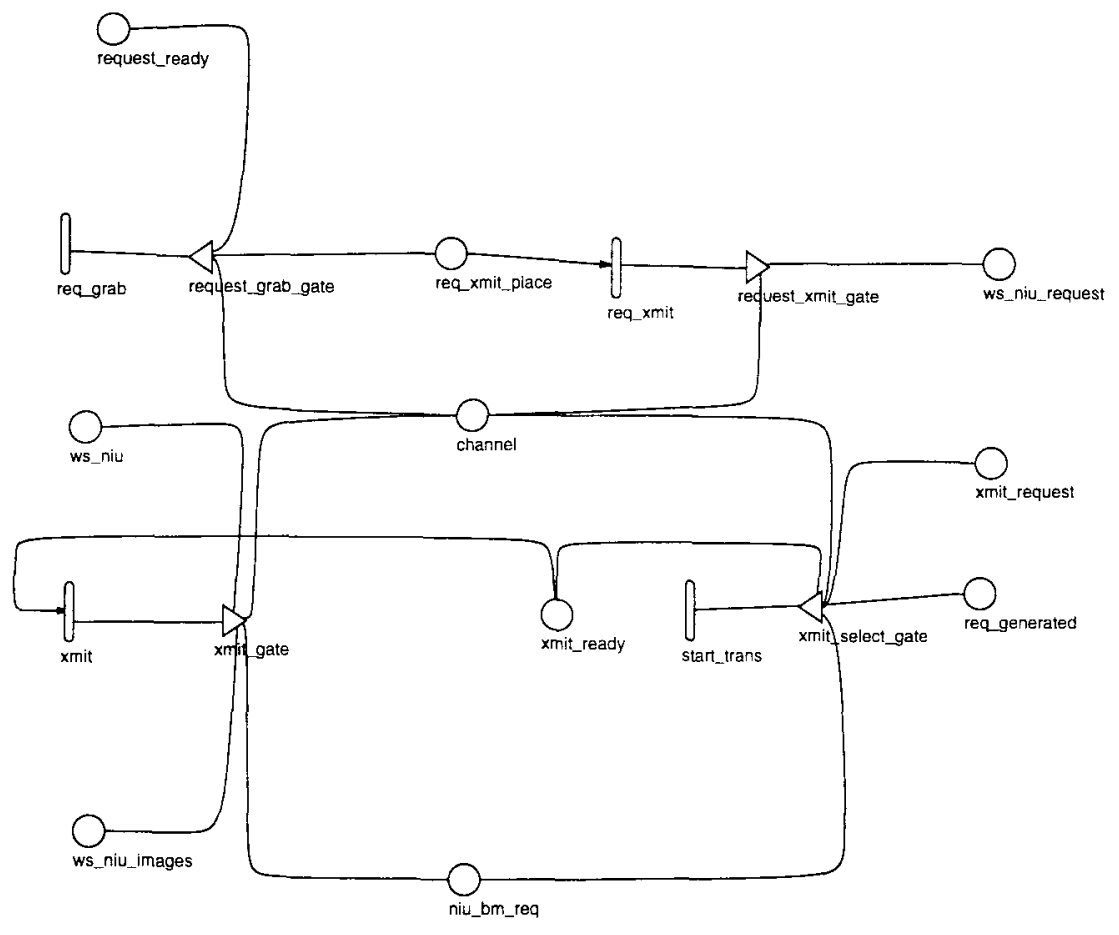

Fig 13. SAN representation of workstation database network interface.

\section{RESULTS}

The described models were simulated using UltraSAN, to estimate various measures of system performance. In particular, we have determined, for the case of two different modality (workload) mixtures and each with a variety of system loads, estimates of the uses of the various resources in the system. We have also estimated performance variables (eg, queue lengths at various points) that are used to determine the response time to a viewing request. All the performance variables specified in the simulations were estimated to a $95 \%$ level of confidence, and error bars are given for each result plotted (in many cases, these error bars are too small to be seen). The system was started cold, ie, empty, and the initial portion of each run was discarded to avoid using the transient period in calculating the estimator. The batch size used was made large enough to assume independence between batch means, and a large enough number of batches was used to avoid problems due to non-normality of the batch samples.

Variation of the system load was accomplished in two different manners. In one case, the number of workstations was fixed at 15 and the viewing time of the images was varied, whereas in the other case, the average viewing time was fixed at 90 seconds and the number of workstations was varied. The patient arrival workload at all imaging modalities was kept fixed, at levels suggested in the American Hospital Association Hospital Technology Series ${ }^{8}$ and given in Table 1. Hardware parameters, such as disk access times and bus speeds, were also fixed at reasonable values. For the case with differing modality mixtures, the following two types were used. In the type-I mix, all three modalities, namely, the MRI, ultrasound and x-ray units were included. In the type-II mix, however, the modality with the highest resource demand (x-ray) was retained while the others were turned off (MRI and ultrasound).

\section{Component Uses}

Figure 14 shows the use of the network and the database CPU versus viewing time of a set of images with 15 workstations. Figure 15 shows the above mentioned uses versus the number of viewing workstations for an average viewing time of 90 seconds.

The figures show that the network use is fairly low for all loads, thereby indicating that the network channel is not the bottleneck in the present PACS environment. The database use 
Fig 14. Database CPU and network uses as a function of viewing time.

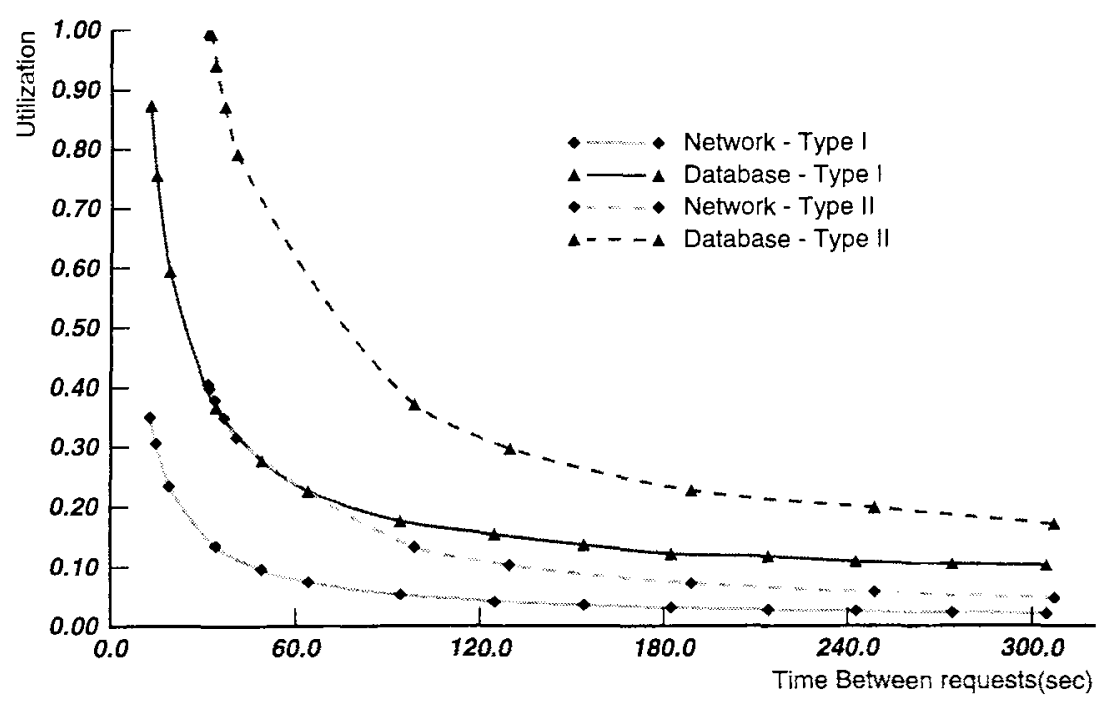

as expected, but the increase for the type-II mix is much steeper. An estimate of these values would prove to be very helpful to a PACS designer, especially in light of widely differing system loads. Finally, for low system loads (viewing times greater than 300 seconds), the database use tapers off to a constant $11 \%$ and $18 \%$ for the case of type-I and type-II modality mixtures, respectively. This use is due to the load of storing new folders from modalities and the subsequent execution of the prefetching algorithm.

Figures 16 and 17 show the hard disk and optical disk uses in the database. As in the previous case, Fig 16 has been used to show the above mentioned uses versus viewing times for

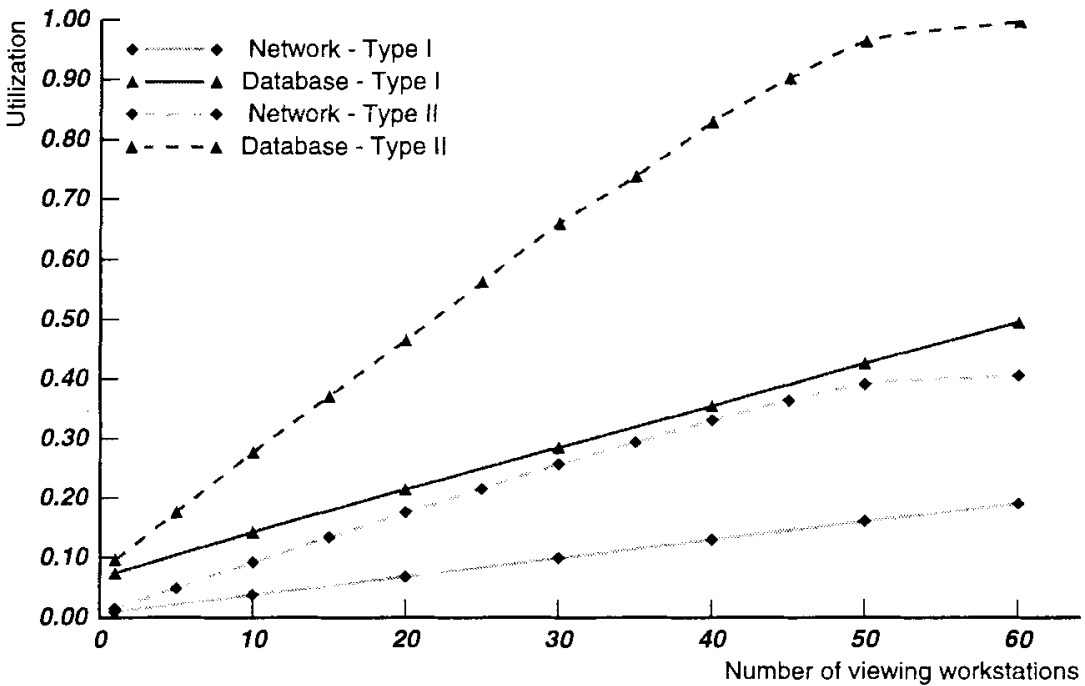

Fig 15. Database CPU and network uses as a function of the number of viewing workstations. 


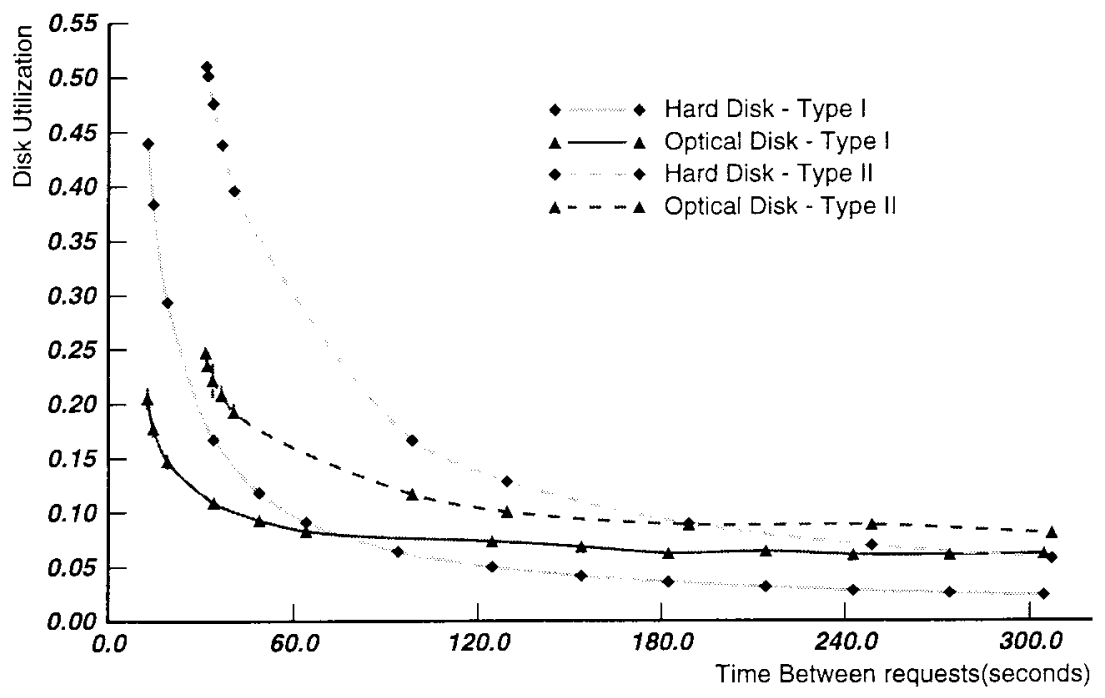

Fig 16. Disk uses in database as a function of the viewing time.

15 workstations, while Fig 17 depicts these uses versus the number of workstations with a fixed viewing time of 90 seconds. From the above figures, it is observed that the hard disk use is lower than the optical disk use for low loads (large viewing times or a small number of workstations). However, as the system load begins to increase, a crossover occurs (at about $8.0 \%$ for the type-I mix and $9.0 \%$ for the type-II mix), after which the hard disk uses exceed those of the optical disk with the difference in magnitude becoming very large for high loads. This sharp disparity at high loads may be attributed to the higher number of read operations on the hard disk, whereas the optical disk is not influenced so strongly (the miss probabil- ity for a request was taken to be .01 ). The prefetch algorithm would affect the optical disk use at higher loads because it would change the miss rate. For lower loads, the hard disk shows very low use whereas the optical disk use tapers off to around $6 \%$ and $8 \%$ for the two modality mixes. Again, this is because of the prefetch algorithms used in the database and because the optical disk access times are much higher than hard disk access times. Finally, as observed before, the disk uses for a given load are higher with the type-II mix than with the type-I mix.

\section{Response Time Calculation}

Figure 18 reports the total response time and the database response time for viewing requests
Fig 17. Disk use in database as a function of the number of viewing workstations.

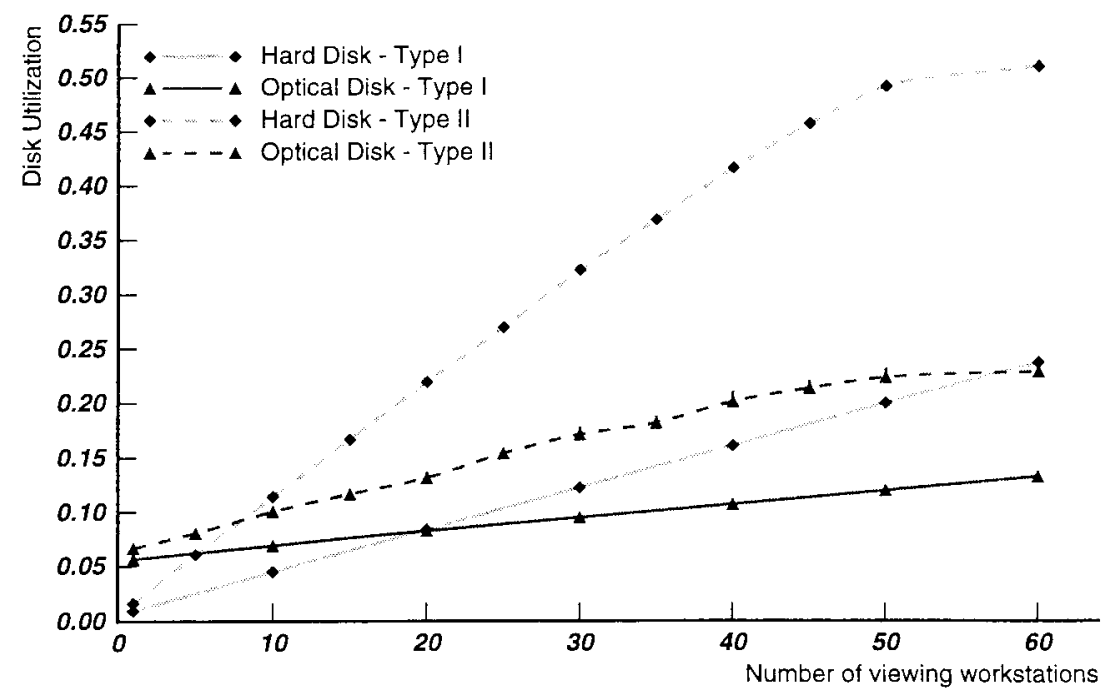


Fig 18. Response time versus

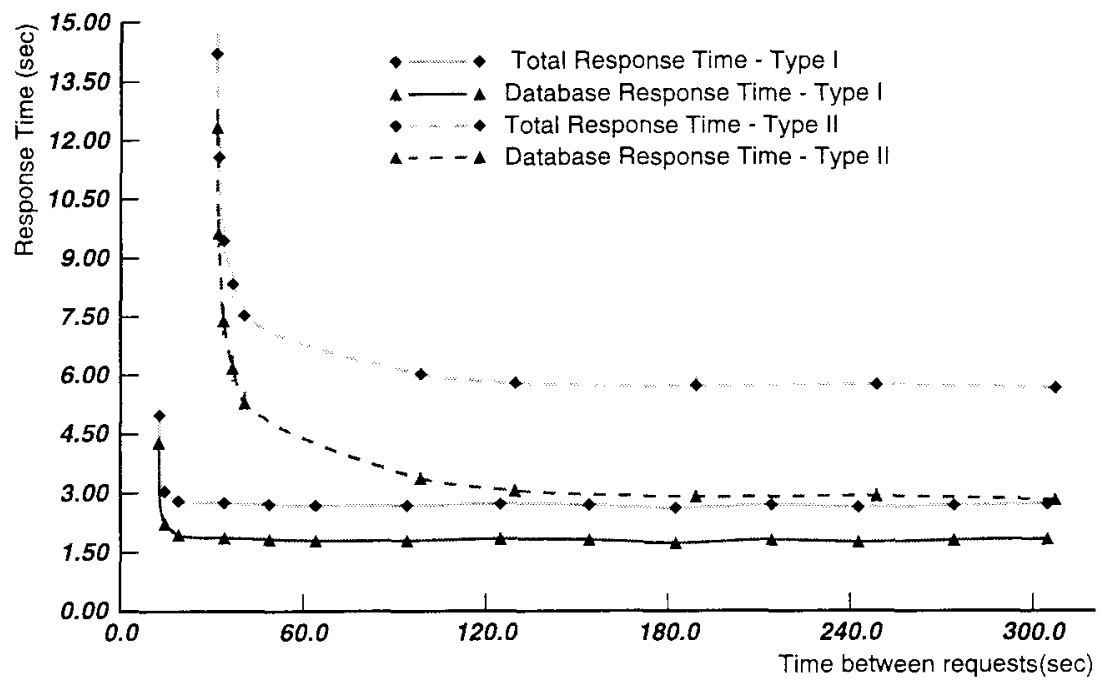

as a function of the image set viewing time, whereas Fig 19 shows the same set of response times as a function of the number of workstations.

The "total response time" in these figures is defined to be the time between the generation of a request for four images and the writing of the first image on the workstation hard disk. Similarly, the "database response time" is the portion of the total response time due to database processing. There are five delay components that contribute to the total response time: (1) delay in the request queue at workstation; (2) transmission delay for a request; (3) delay at the database due to retrieval time; (4) transmission delay for the retrieved image; and (5) delay due to writing the image to hard disk at the workstation.

The above mentioned delay components may be calculated using Little's result, ${ }^{11}$ as shown in Fig 20. Boxes $B$ through $E$ are seen to contain a set of places that are present in the SAN model of the PACS. These places represent either a queue or a server. The arrival rate of tokens into all places in a box is the same. Little's result can be applied to each individual box and the total expected delay can be obtained by summing these partial delays. The rate in box $A$ is the rate at which the viewing images are requested. Because each request token in box $A$ represents a request for 4 images, the effective rate into the other boxes $B$ through $E$, is four times this rate.

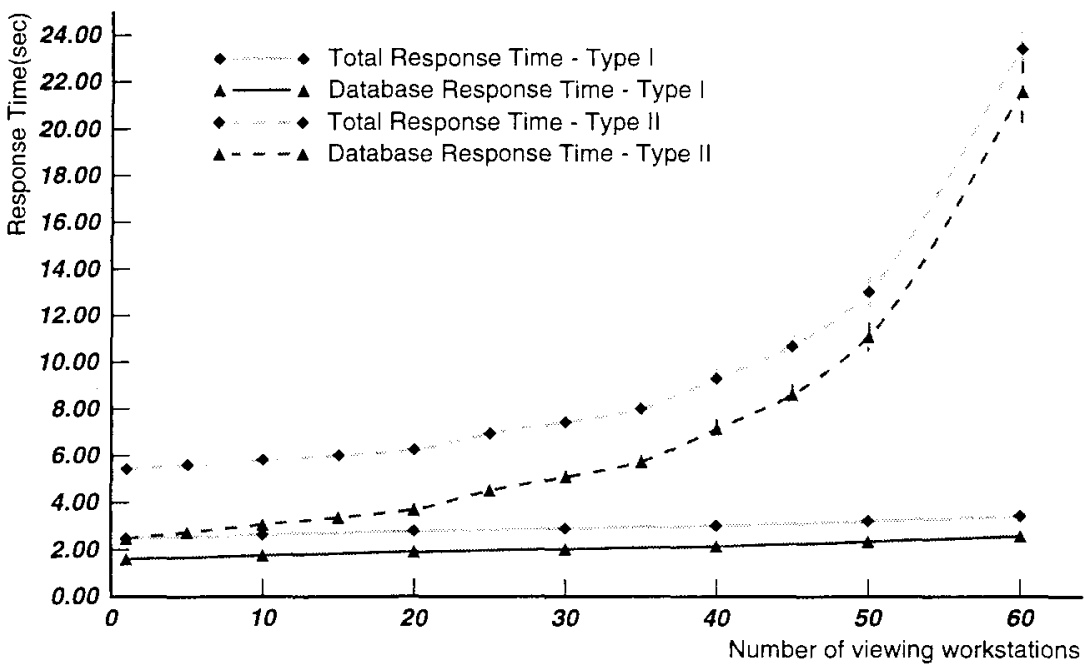

Fig 19. Response time versus number of viewing workstations. 
BOX A

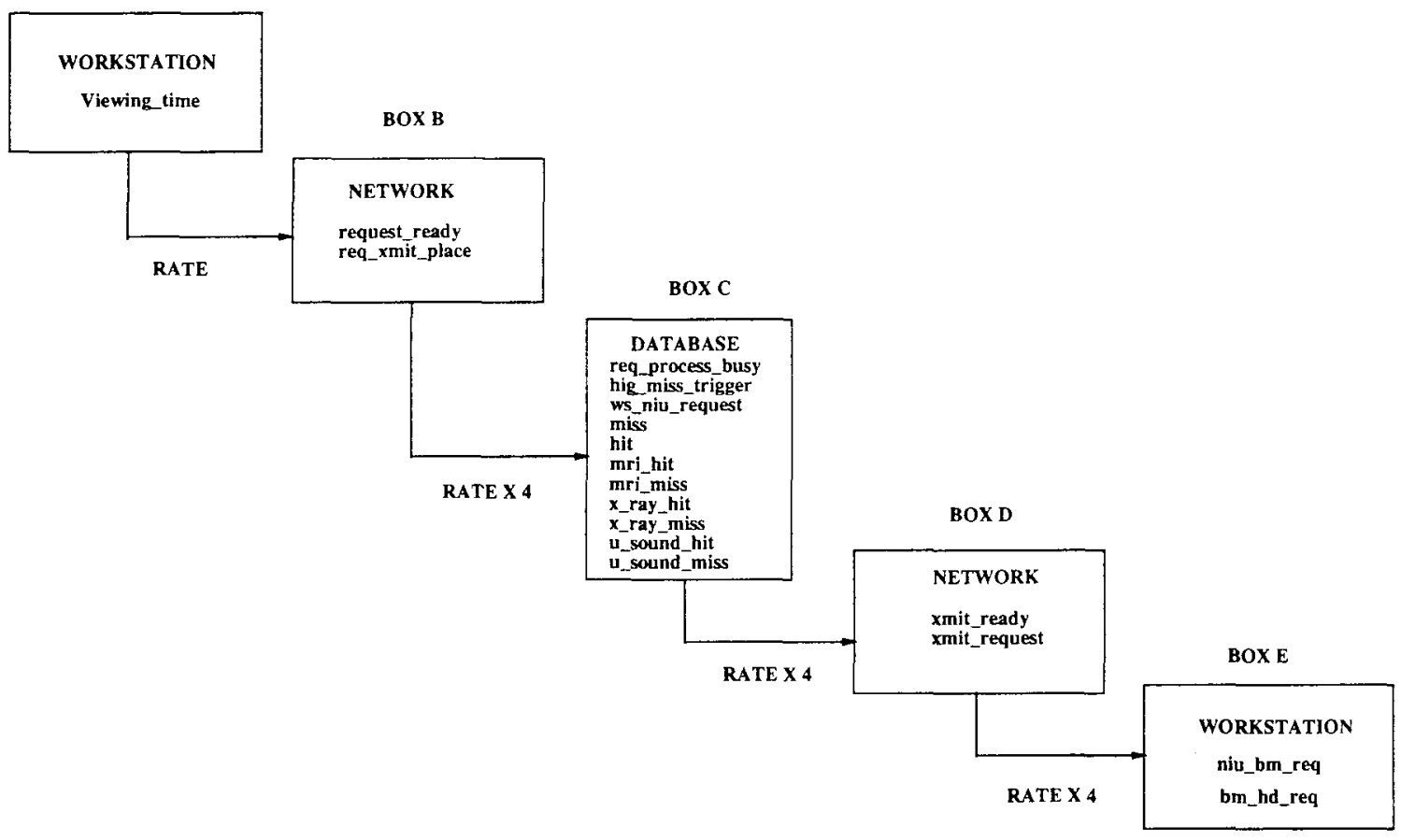

Fig 20. Response time calculation using Little's result.

Box $B$ is used to compute the delay involved in the transmission of a request token. The delay in the database is calculated with the help of box $C$, while boxes $D$ and $E$ respectively compute the image transmission delay and the delay in the workstation after the image has been received.

From the total response time curve, we observe that the delay remains fairly low and constant under a wide range of loads for both types of modality mixtures. In fact, for the type-I mix (wherein we have all the three types of modalities present), the response times are low until a viewing time of just 10 seconds between requests, indicative of a fairly long "stable operation portion" with a low and constant delay. The delays for type-II mix, however, are larger than their counterparts for the type-I mix, which, as observed before, is because all of the images requested in this set require a considerable (in fact, the maximum) amount of system resources. Furthermore, the "low and constant" portion of the delay curve in this case is seen to break away at around the 60 seconds zone, which is earlier than the type-I mix case. Thus, depending on the modality mix, an estimate of the total delay for the given PACS at low system loads is seen to be around 2.6 and 5.6 seconds for the two widely differing modality mix cases studied in the current work. The stable operation is seen to extend to 10 seconds in one case and 60 seconds in the other, after which the delays, under the increasing system loads, begin to increase sharply just as expected. Thus the ideal operating region for the PACS would be in the flat portion of the response time curve. The database response time curve essentially follows that of the total response time, indicating that the database is causing the increase in response time for higher system loads. The reason for this is evident from the use curves for the database that were discussed earlier. Clearly, a faster database CPU and faster hard disk access times in the database would improve the performance of the PACS.

Thus, these models and results show that SANs provide a suitable modeling framework to evaluate the performance of PACS. Heirarchical (composed) models of the various PACS components may be constructed, and the performance of the composed model may be evalu- 
ated and bottlenecks identified. The effect of varying system parameters on the overall performance of a given PACS configuration may also be examined, as was done in the current work. Furthermore, while the assumptions made in modeling this particular PACS do not apply to all PACS, they show how to construct SAN models of PACS given assumptions reasonable for a particular installation. Different assumptions would lead to different SAN models, without a great change in the complexity of the model. Hence, this work shows how SANs and composed models can be used to construct and evaluate a particular PACS configuration as dictated by the application on hand, along with the desired system parameters.

\section{SUMMARY}

PACS are expected to have a high impact on the efficiency of hospital management systems. Currently, most of the radiological image processing in hospitals is manual, which limits the speed and efficiency of processing. Upgrading to a completely automated electronic system would be an expensive process in terms of equipment and software costs. Thus, the modeling and simulation of PACS is a relatively inexpensive approach to obtaining performance estimates for different system configurations before actually upgrading the hospital with a PACS. These performance estimates help trim down the system size and hence the cost. In addition, these estimates can help in identifying system bottlenecks so that more effort can be channeled into reducing the bottlenecks.

For the particular PACS studied in this article, simulation studies have indicated that the performance bottleneck in the system is the database and that the database and overall performance can be improved by having a faster database processor and hard disk clusters with faster access times. Performance curves were obtained for component uses in the database (hard disk and optical disk) and for response times to image viewing requests made at the workstations. Finally, the performance of the PACS system was examined under the influence of a widely differing modality mix including the performance of the system in the presence of a single modality type with the heaviest workload demand.

The results and models developed also show that SANs are a modular and efficient modeling formalism that can be effectively used to evaluate PACS. PACS tend to be large with many different types of imaging modalities, databases, and workstations, and many units of each type. Reduced base model construction makes the modeling process for such systems with identifiable physical and logical subunits easy. Large models can be constructed in a hierarchical fashion using the construction operations discussed in this work.

\section{REFERENCES}

1. Stut WJJ Jr, van Steen MR, Groenewegen LPJ, et al: Picture archiving and communication system design issues: The importance of modelling and simulation. J Digital Imaging 3:246-253, 1990

2. Bakker AR, Didden H, de Valk JPJ, et al: Considerations on an algorithm for activation of images in a multilayered storage system within a PACS. Proc SPIE PACS IV 626, 1986

3. Movaghar A, Meyer JF: Performability modeling with stochastic activity networks. Proc 1984 Real-Time Systems Symp, Austin, TX, December 1984, IEEE Computer Society Press

4. Meyer JF, Movaghar A, Sanders WH: Stochastic activity networks: Structure, behaviour and application. Proc Int Workshop on Timed Petri Nets, Torino, Italy 106-115, July 1985, IEEE Computer Society Press

5. Sanders WH, Meyer JF: Reduced base model construction methods for stochastic activity networks. IEEE Journal on Selected Areas in Communications 9:25-36, 1991
6. Couvillion J, Freire R, Johnson R, et al: Performability modeling with UltraSAN. IEEE Software 8:69-80, 1991

7. Sanders WH, Freire RS: Efficient simulation of hierarchical stochastic activity network models. Discrete Event Dynamic Systems: Theory and Applications 3:271-300, 1993

8. American Hospital Association Hospital Technology Series (ISSN 0888.711X), Guideline Report, 1989

9. Sanders WH, Martinez R, Alsafadi Y, et al: Performance evaluation of a picture archiving and communication network using stochastic activity networks. IEEE Trans Med Imaging 12(1), 1993

10. Sanders WH, Kant L, Kudrimoti A: A modular method for evaluating the performance of picture archiving and communication systems. Performability Modeling Research Laboratory Technical Report, The University of Arizona, January 1993

11. Little JDC: A proof of the queueing formula $L=\lambda \mathrm{W}$. Operations Research 9:383-387, 1961 